\title{
The circuit design for extraction of electro encephalogram signals
}

\author{
LIU Wenqiang1, QIU Lijun², FAN Qifu', LIU Huan ${ }^{4}$ \\ College of control engineering, Xijing University, Xi'an 710123 \\ E-mail: 1 liu-wq-66@163.com ; 2 qiulij@fmmu.edu.cn
}

Keywords: EEG signals; amplification; Wave filtering; Circuit Design

Abstract: The design of front-end signals acquisition circuit is made for extraction of EEG signals, thus making it possible to amplify, filter, and remove the interference of source signal collected from the brain electrode. After the signal go in turn through such links as pre-amplification, high-pass filtration, $50 \mathrm{~Hz}$ wave trapping, low-pass filtration, and post amplification, the EEG signals average amplitude of $50 \mu \mathrm{V}$ can reach to between $1 \mathrm{~V} \sim 4 \mathrm{~V}$, thereby meeting the range of requirements the conventional acquisition card needs for the input of analog signal. The simulation results show that the signal, as collected through the circuit, are low in distortion, and thus can be widely used in the devices for processing EEG signals.

\section{Introduction}

EEG signals is produced spontaneously by the brain nerve electrical activity existing in the central nervous system and having rich brain activity information, through the detection and extraction of EEG signals, except for brain research, clinical brain disorders, but also can be used as a sleep quantitative monitoring [1]. High quality EEG signals can provide clinical data diagnosis, and as a noninvasive diagnostic detection methods. It has the extremely vital significance in clinical diagnosis and treatment of encephalopathy in the brain cognitive function research and development [2].

At present, the circuit structure of pre-amplifier phase parallel is designed by American O'Brient company [3]. At home, EEG signals pre-amplifier circuit was designed composed of two instrumentation amplifier by Sanqiang Wang [2], Shiwei Ma designed a three magnified three filtering EEG acquisition circuit [4], Youming Sun designed a amplifier circuit of EEG signals based on three op-amp[1].

\section{Circuit design}

EEG signals is signal of low frequency, weak and strong noise background, which is an unstable and nature signal issued by the complex life [5]. It is between $10 \mathrm{~V} \sim 100 \mathrm{~V}$, while the input signal to noise ratio is up to 1:10000; brain electrical signal frequency of EEG signals is low, its range is generally from $0.5 \mathrm{~Hz} \sim 35 \mathrm{~Hz}[2]$, which makes the amplifier low cut-off frequency selection is very difficult. When subjected to sharp pulse disturbance or lead switch, amplifier is prone to clog, EEG signal is vulnerable in the environment during the process of extraction, this will cause the signal distortion. The special electrode EEG signals extraction signal by the differential amplifier processing and transmission and the amplifier for receiver can display and analysis of EEG signals by oscilloscope or USB interface in the computer.

In order to overcome the shortages of the existing technology and to meet the requirements of EEG signals acquisition technology, we design the effective detection and extraction of EEG signals based on the existing technology of the detection circuit. The circuit structure of the module block diagram shown in figure 1. 


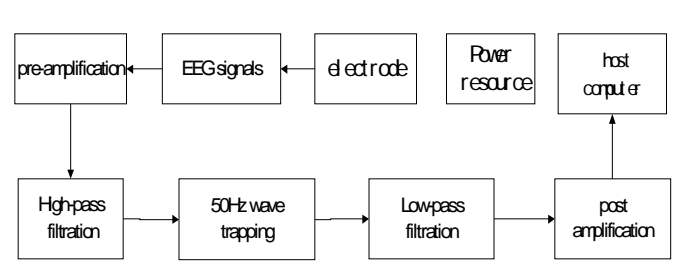

Fig 1 the circuit structure of the module block diagram

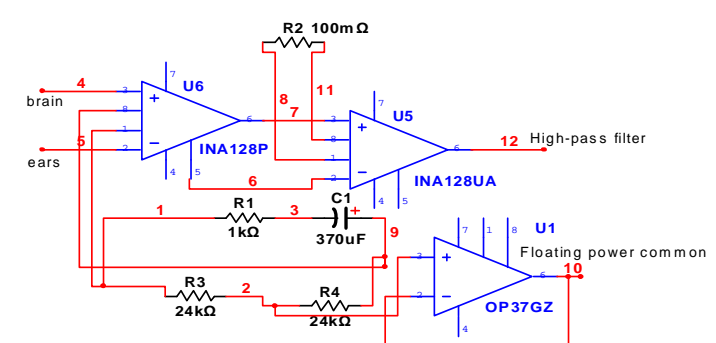

Fig 2 the design of pre-amplifier circuit diagram

\section{The pre-stage main amplifier}

In such a multistage amplifier circuit, design of pre-amplification circuit is a key link in the EEG signals data acquisition system. In order to design a high quality of EEG signals amplifier, the pre-amplifier must have high input impedance, high common mode rejection ratio, low noise, small non-linearity, strong anti-interference ability, the appropriate frequency and the range of dynamic [3], which makes the design of amplifier that is very difficult, but it is the most important to design circuit of acquisition the total system EEG signals.

The design based on two paralleling INA128 instrumentation amplifier is improved by literature 2, the essence is two in-phase parallel structure of the preamplifier circuit cascade. The purpose is to improve the zero drift and common mode rejection ratio of the system circuit. The circuit principle diagram is shown in figure 2.

The times magnification of the circuit: (refer with: Eq. 1)

$$
A_{d}=A_{1 d} \times A_{2 d}=\left(1+\frac{50}{R_{1}}\right)\left(1+\frac{50}{R_{2}}\right) A_{d}=25551
$$

Common mode rejection ratio: (refer with: Eq. 2)

$$
C M R R=C M R R_{1} \times C M R R_{2}=\frac{A_{1 d}}{A_{1 c}} \times \frac{A_{2 d}}{A_{2 c}}
$$

Voltage gain:(refer with:Eq.3)

$$
G \text { a in }=10+10 \times \frac{R_{1}}{R_{2}}=110
$$

$\mathrm{CMRR}_{1}$ and $\mathrm{CMRR}_{2}$ are the primary circuit and the two circuit of common mode rejection ratio, $A_{1 d}, A_{1 c}, A_{2 d}$ and $A_{2 c}$ are respectively the primary circuit and the two circuit differential mode gain and common mode gain. Due to the EEG signals is easily affected by interference of the power frequency and the radio frequency, etc, these signals are easily into input through pins 4 and 5 the brain electrode in the figure 1, which can be suppressed by improving the amplifier resistance. Designed by the DC blocking capacitor and connected in series to form a capacitive resistance coupled circuit is designed to the interference of suppress [2]. EEG signals the frequency of not less than $0.5 \mathrm{~Hz}$, and $f=\frac{1}{2 \pi R C}$ in the RC circuit, selection $R=1 k \Omega, C=330 \mu \mathrm{F}$, floating track circuit is designed to further improve the common mode rejection ratio of pre-amplifier circuit [3], the circuit magnification is up to 25551 times. For INA128, the voltage gain of resistance when access to pins 1 and 8 more than $100 \mathrm{~dB}$, common mode rejection ratio is greater than $120 \mathrm{~dB}$ [1], the circuit is up to $200 \mathrm{~dB}$, to meet the EEG of common mode rejection ratio of not less than the requirements of $120 \mathrm{~dB}$.

\section{The high pass-filtration}

Design of a high-pass filtration is to filter the DC component in the circuit, we design the volume resistance coupled circuit, the RC circuit and prevent base-line drift. In the RC circuit, the more product of RC, the better low frequency of high-pass filtration. Because of lower frequency of EEG 0.5 $\mathrm{Hz}$, in order not to lose their low frequency components, the filter cut-off frequency is set to $0.3 \mathrm{~Hz}$. So the product of $\mathrm{RC}$ can meet the requirements for 2.78 . The circuit principle diagram is shown in figure 3. 


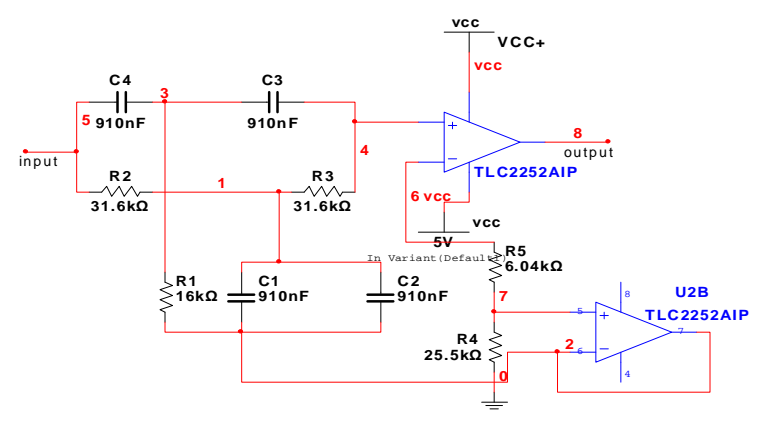

Fig 3 the design of high-pass filter circuit diagram

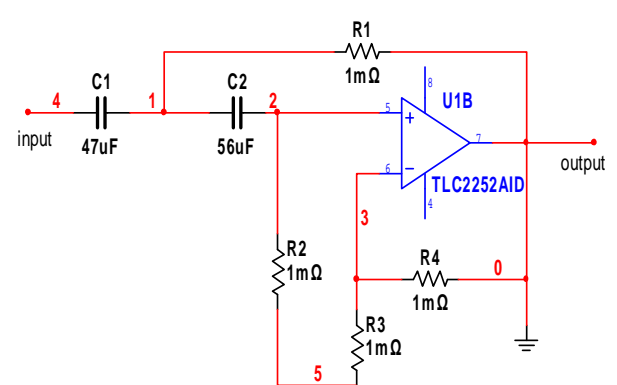

Fig 4 the design of $50 \mathrm{~Hz}$ band-pass filter circuit diagram

\section{The $50 \mathrm{~Hz}$ band-pass filter}

In the non-shielded condition $50 \mathrm{~Hz}$ power frequency is the main interference in EEG signals. in the pre-amplifier circuit of the common mode interference in the form of a strong inhibition, but also to the differential mode signal interference into the main circuit [2]. It must be through the filter to remove the disturbance of $50 \mathrm{~Hz}$ usually adopts the band stop filter. The circuit principle diagram is shown in figure 4.

\section{The Low-pass filtration}

Low-pass filter undertakes double tasks before to inhibit a broad spectrum of noise and Analog-to-Digital Converter of anti aliasing, namely through the low-frequency signal, attenuate or suppress the high-frequency signal [1]. In order to obtain a high signal to noise ratio, both of them require low-pass filter pass-band as smooth as possible, roll rate as soon as possible. In order to make the filtration more close to ideal low-pass filtration, the Butterworth filtration of the system, namely cascaded filter by a double of two order voltage controlled active low-pass filter. According to the needs of design, the research of EEG signals for low-frequency signal, the experimental use of the general to the filter cut-off frequency is set to $56 \mathrm{~Hz}$, to filter out high-frequency noise, and EEG signals is maximum reserved. The circuit principle diagram is shown in figure 5.

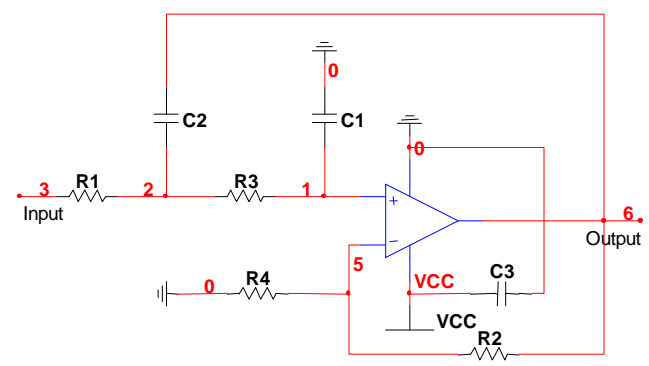

Fig 5 the design of low-pass filter circuit diagram

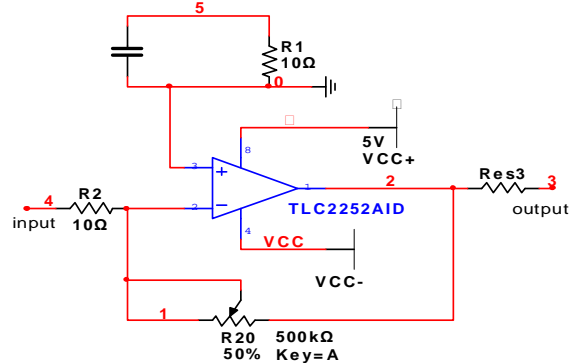

Fig 6 the design of post-stage amplification circuit diagram

\section{The post-stage amplification}

The voltage range of the pre-amplifier circuit of the EEG signals is still in $\mathrm{mV}$ level, also need to post-stage amplifying circuit is further amplified. The system total magnification 20000 100000 times, the post-stage circuit only amplified 5 10 times can meet the requirements after the pre-magnified 20000 times. Therefore, it is designed to adjustable gain to improve the practicality of the system. The reverse amplifier, and voltage gain by adjust potentiometer. In addition, the operational amplifier has zero drift and offset voltage [4], which has adverse effects to detecting EEG signals. Therefore, the offset voltage and the zero drift are adjusted to zero. Schematic diagram of Circuit is shown in figure 6.

\section{Circuit simulation and debugging}

In the design process, we use Multisim12.0 software pre-amplifier module for circuit simulation debugging. When the whole circuit magnification is 25000 , the simulation results: the amplitude of the input signal (Figure 7) of $50 \mu \mathrm{V}$, frequency of $50 \mathrm{~Hz}$, the output signal amplitude of about $1.5 \mathrm{~V}$ (Figure 8).The simulation results are shown in Figure 9, EEG signals average amplitude of $50 \mu \mathrm{V}$ is amplified amplitude can reach to $1 \mathrm{~V} \sim 4 \mathrm{~V}$, which can meet requirements of the conventional acquisition card for analog signals. But further inhibited the baseline drift, provide true analog EEG signals for receiving 
end function such as the USB interface or oscilloscope can EEG signals in the computer display and characteristic analysis.

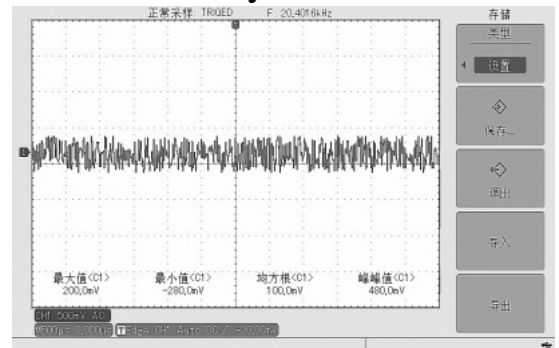

Fig 7 input signal

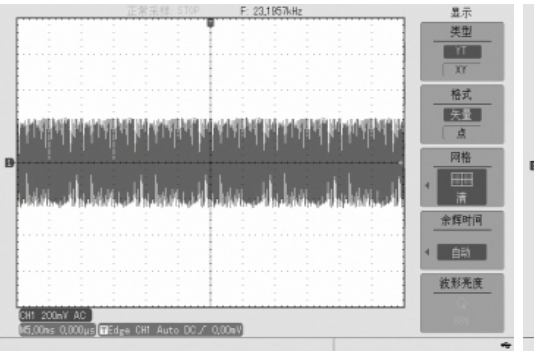

Fig 8 primary signal amplification

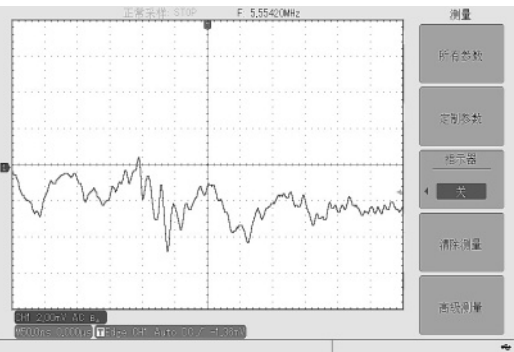

Fig 9 output signal

\section{Conclusion}

We designed the acquisition and preprocessing EEG signals of two-stage amplifying and three-stage filtering circuit, EEG signals detection and feature extraction circuit integrated devices, to solve the $50 \mathrm{~Hz}$ power frequency interference, high common mode rejection ratio, strong anti-interference. Has the circuit debugging convenient collection effect is good, which can meet the requirements of the conventional acquisition card for analog signal input range. The circuit has the advantages of simple structure, stable performance. In addition, in the selection of circuit components on the performance of new products with excellent, improved the reliability of the circuit performance. In the future, EEG detection will greatly popular in daily life, family, health, basic education field will be used widely, so it has great potential advantage.

\section{Acknowledgements}

This work was financially supported by the science foundation research project of Xijing University (Grant No. XJ150112), the nature science foundation research project of Shaanxi province (Grant No. 2014JM4176) and the nature science foundation research project of Shaanxi province (Grant No. 2016JM1032).

\section{References}

[1] Youming Sun, Binglian Huang, Xiaoshu Luo. A new model of EEG signal design of amplification and detection circuit [J]. Medical Equipment. 2007 (1).p.15-17 (in Chinese)

[2] Sanqiang Wang, Wei He, Jian Shi. Model of EEG signals pre-amplifying circuit design [J].Journal of Chongqing University (Nature science edition) 2006 (6) p.51-53 (in Chinese)

[3] Shiwei Ma, Junqiang Guan, Junqiang Yang, Ling Yuan. BCI for the EEG signals detection circuit design of measurement and control technology [J]. 2009 (6). p.28-31 (in Chinese)

[4] Zhencheng Chen, Jing Zhong. Cerebral electrical signal acquisition pre-conditioning circuit design [J]. Journal of medical physics China. 2009 (7). p.1299-1301 (in Chinese)

[5] Jinzhi Zhou, etc. Based on MSP430 development and application of embedded system [M]. Chemical Industry Press. 2013.7.p.195-198 (in Chinese)

[6] Liu Jun, Binzhen Zhang. Weak signals detection technology [M]. Electronic Industry Press .2005. (10). p.245-257 (in Chinese)

[7] Yang Yang. Design and Research. EEG signals acquisition and analysis system [D]. Hefei University of Technology .2006.(7). p.135-148 (in Chinese)

[8] Shaohua Yin, Jihai Yang, etc. Based on quantitative analysis of surface electromyography recursive feature extraction and classification [J]. University of Science and Technology of China. 2006, 36 (5) .p. 34-41 (in Chinese) 
[9] Angelone LM, Potthast A, Segonne F, et al. Metallic electrodes and leads in simultaneous EEG -fMRI: a specific absorption rate (SAR) simulation studies[J]. Bioelectromagnetics, 2004(4) .p.285-295. 